\title{
Imaging Spectrum of Lobular Carcinoma In Situ and Correlation with Pathology Findings
}

\author{
Pradipta C Hande ${ }^{1}$ Sarabjeet Kaur Arneja ${ }^{2}$ Sabita S Desai ${ }^{3}$
}

\author{
${ }^{1}$ Department of Radiology and Imaging, Breach Candy Hospital Trust, \\ Bhulabhai Desai Road, Mumbai, Maharashtra, India \\ 2 Department of Surgical Pathology and Cytology, Breach Candy \\ Hospital Trust, Bhulabhai Desai Road, Mumbai, Maharashtra, India \\ ${ }^{3}$ Department of Radiology, Breach Candy Hospital Trust, Bhulabhai \\ Desai Road, Mumbai, Maharashtra, India
}

\author{
Address for correspondence Pradipta C Hande, MD, Department of \\ Imaging, Breach Candy Hospital Trust, Bhulabhai Desai Road, \\ Mumbai, Maharashtra, 400 026, India \\ (e-mail: pradipta.hande@gmail.com).
}

Indian J Radiol Imaging 2021;31:551-559.

\begin{abstract}
Keywords

- architectural distortion

- digital breast tomosynthesis

- hook-wire localization

- immunohistochemistry

- lobular carcinoma in situ

- microcalcifications

Background Lobular carcinoma in situ (LCIS) is a noninvasive neoplasm that is known to have an increased relative risk for developing subsequent invasive breast carcinoma. Pure LCIS is usually an incidental finding on histopathological examination (HPE) of tissue samples. However, in the recent years, there has been an increasing trend seen in the diagnosis of LCIS.

Purpose This article aims to bring out the spectrum of appearances on breast imaging in confirmed cases of pure LCIS on HPE and immunohistochemical.

Materials and Methods Cases that were confirmed as pure LCIS on HPE from core or excision biopsy were retrospectively analyzed for abnormalities on breast imaging. Digital breast tomosynthesis mammography was performed with high-resolution ultrasound with elastography for all cases. Magnetic resonance imaging (MRI) was performed in cases wherever indicated, with dynamic postcontrast imaging after injecting intravenous gadolinium.

Conclusion LCIS is recognized as an intermediate risk factor for the development of breast cancer. Pure LCIS has varied histology and imaging patterns on mammography, high-resolution ultrasound, and MRI. It is important to recognize the imaging appearances of these lesions to enable the radiologist to detect LCIS early for proper management.
\end{abstract}

\section{Introduction}

Early detection of breast cancer with radiological screening has resulted in early treatment and management which has increased survival.

Lobular carcinoma in situ (LCIS), is a noninvasive proliferation of lobular cells within a terminal-duct lobular unit (TDLU). ${ }^{1}$ Atypical lesions and LCIS are associated with an increased risk of breast malignancy. ${ }^{1,2}$ These women have 7 to 12 times increased relative risk of developing invasive breast carcinoma after the diagnosis of LCIS. ${ }^{3}$ The true incidence of LCIS is difficult to estimate, as classic LCIS (cLCIS) is rarely detected on conventional mammograms and is usually an incidental finding in a breast biopsy or specimen performed for other abnormalities. ${ }^{4,5}$

In the recent years, there is an increased incidence of LCIS seen which is likely due to the increased screening for breast
DOI https://doi.org/ 10.1055/s-0041-1734411. ISSN 0971-3026.

\footnotetext{
(C) 2021. Indian Radiological Association. All rights reserved. This is an open access article published by Thieme under the terms of the Creative Commons Attribution-NonDerivative-NonCommercial-License, permitting copying and reproduction so long as the original work is given appropriate credit. Contents may not be used for commercial purposes, or adapted, remixed, transformed or built upon. (https://creativecommons.org/ licenses/by-nc-nd/4.0/) Thieme Medical and Scientific Publishers Pvt. Ltd., A-12, 2nd Floor, Sector 2, Noida-201301 UP, India
} 
cancer with better imaging techniques. Standardized breast imaging reporting using breast imaging reporting and data system (BI-RADS) lexicon and further needle biopsy of indeterminate or suspicious lesions with subsequent surgical excision has contributed to this trend. ${ }^{1}$

The purpose of this article is to describe and assess the imaging findings of LCIS on mammograms and/or supplemental modalities as high-resolution ultrasonography (USG) and contrast-enhanced magnetic resonance imaging (CEMRI) in correlation of with histopathological examination (HPE) including immunohistochemistry (IHC).

\section{Materials and Methods}

\section{Study Design}

This was a hospital-based retrospective observational study.

\section{Study Population}

\section{Inclusion Criteria}

All cases that were confirmed as LCIS on pathologic examination on core biopsy or surgical excision of the lesion and had abnormalities on breast imaging were included.

Women with no symptoms underwent screening mammography as part of annual health checkup. Diagnostic mammography was performed for patients who presented with clinically suspected breast lesions.

\section{Study Duration}

72 months from January 1, 2014 to December 31, 2019.

Imaging techniques:

- In our institution, digital breast tomosynthesis (DBT) and fullfield digital mammography system (FFDM) is done on Selenia Dimension HD machine (Hologic , Inc. Bedford, Mass, USA.). Reconstructed $1 \mathrm{~mm}$ thin-slice images are studied in conjunction with two dimensional (2D) synthesized mammography (2DSM; C-view) images that are obtained from the DBT dataset. Routine mediolateral-oblique and craniocaudal views were obtained for both breasts. Women underwent opportunistic screening mammography as part of health checkup. Diagnostic mammography was performed for patients who presented with clinically suspected breast lesions. Additional spot compression/magnification views were done when necessary

- Supplemental USG on Acuson S2000 (Siemens Healthcare $\mathrm{GmBH}$, Germany) machines were performed by the radiologist in all cases using high-resolution 11 to $12 \mathrm{MHz}$ transducer

- MRI was performed on 3 Tesla MAGNETOM Vida MRI scanner (Siemens Healthcare GmBH, Germany) with 16channel breast coil was used

- Patient preparation:All metallic subjects were far from the patient body including zippers and clasps. Intravenous line was established for gadolinium injection

- Patient positioning:The patient was prone with breasts positioned onto the breast coil, with the nipple faced straight down. The patient was instructed not to move during the scan.
- Imaging protocol:After localizers, axial T2-weighted-turbo spin echo sequence (DIXON), short tau inversion recovery sequence, precontrast plain axial, and coronal T1-weighted (T1W) images were acquired. Dynamic fatsuppressed high-resolution TIW 3D fast gradient-echo images were sequentially acquired after administration of intravenous contrast gadolinium at a dose of 0.1 $\mathrm{mmol} / \mathrm{kg}$ of body weight with $2 \mathrm{~mL} / \mathrm{s}$ flow rate with saline flush using a power injector. Postcontrast images are acquired at 60,120,180,240, and 300 seconds.

- MRI contrast:Gadolinium dimeglumine (Magnaview, Magnus, India).

\section{Image Interpretation}

Mammograms in all the cases were analyzed for the presence of masses, architectural distortion, asymmetry, and calcifications. The detected microcalcifications were described further depending on their types and distribution.

The ACR BI-RADS Atlas, Fifth Edition, was followed while describing the imaging findings categories on mammography and USG. ${ }^{6}$ The lesions included in the article were mostly assigned BI-RADS category 4 (A, B, or C) or 5 and biopsy was recommended. In four (16\%) cases, category BI-RADS 0 was assigned.

Eighteen cases underwent adjunctive CEMRI as recommended by the surgeon or clinician. Lesion morphology with mass or nonmass enhancement characteristics was assessed along with dynamic contrast-enhanced images with quantitative kinetic curves. A fast pattern indicated an increase in intensity of $>100 \%$ as compared with a slow pattern with an increased intensity of $<50 \%$ and a medium pattern between 50 and $100 \%$. The delayed phase kinetic feature was categorized as persistent, plateau, or washout. ${ }^{3}$

Suspicious lesions detected on imaging that warranted biopsy, targeted breast USG for USG-guided biopsy. If no lesion was seen on USG, stereotactic biopsy or surgical excision was performed with needle localization under mammographic guidance.

G Biopsy needle (Bard) is usually used for USG-guided biopsy in our department. Stereotactic mammography-guided biopsy was done for cases with microcalcifications using Magnum biopsy gun (Bard) with disposable needle. Imageguided preoperative hook-wire localization using Kopans needle was done on USG or mammography as suitable for a particular case. Specimen radiography was also obtained to confirm the removal of the lesion. Imaging findings were then correlated with the pathological findings that were confirmed as LCIS and included as the cases for the study.

\section{Observations and Results}

Forty-one cases were diagnosed as LCIS on HPE of tissue biopsy or on surgical excision for suspicious abnormalities on breast imaging. Twenty-eight asymptomatic women came for screening mammography and 13 presented with 
Table 1 Abnormalities detected on digital mammography/ digital breast tomosynthesis

\begin{tabular}{|l|l|l|}
\hline Abnormality & $\begin{array}{l}\text { Cases } \\
(\boldsymbol{n}=\mathbf{2 5})\end{array}$ & $\%$ \\
\hline Microcalcifications & 6 & 24 \\
\hline Mass & 3 & 12 \\
\hline Mass with calcifications & 3 & 12 \\
\hline Asymmetry & 3 & 12 \\
\hline Focal asymmetry and calcifications & 4 & 16 \\
\hline Architectural distortion & 3 & 12 \\
\hline $\begin{array}{l}\text { Architectural distortion and } \\
\text { microcalcifications }\end{array}$ & 2 & 8 \\
\hline Abnormal ducts & 1 & 4 \\
\hline
\end{tabular}

Table 2 Salient features of abnormalities detected on breast imaging

\begin{tabular}{|c|c|}
\hline Abnormality & Cases \\
\hline Mammography $(n=25)$ & $n(\%)$ \\
\hline Mass only & $3(12)$ \\
\hline Microcalcifications only & $6(24)$ \\
\hline Architectural distortion (AD) only & $3(12)$ \\
\hline Asymmetry, focal asymmetry (FA) & $3(12)$ \\
\hline Dilated ducts only & $1(4)$ \\
\hline Mass with microcalcifications & $3(12)$ \\
\hline AD with microcalcifications & $2(8)$ \\
\hline FA with microcalcifications & $4(16)$ \\
\hline \multicolumn{2}{|l|}{ Ultrasound $(n=25)$} \\
\hline Mass-hypoechoic, irregular margins & $3(12)$ \\
\hline Mass-well circumscribed, oval & $3(12)$ \\
\hline Dilated ducts with echogenic lesion & $1(4)$ \\
\hline Hypoechoic architectural distortion & $2(8)$ \\
\hline Hypoechoic lesion, indistinct margins & $3(12)$ \\
\hline $\begin{array}{l}\text { Heterogeneous hypoechoic area, } \\
\text { calcific foci }\end{array}$ & $2(8)$ \\
\hline Negative findings & $11(44)$ \\
\hline Axillary lymphadenopathy associated & $2(8)$ \\
\hline \multicolumn{2}{|l|}{$\begin{array}{l}\text { Contrast-enhanced magnetic } \\
\text { resonance imaging }(n=18)\end{array}$} \\
\hline Focus/foci with enhancement & $5(27.7)$ \\
\hline Enhancing oval mass & $1(5.5)$ \\
\hline Enhancing irregular mass & $3(16.6)$ \\
\hline Nonmass enhancement & $8(44.4)$ \\
\hline $\begin{array}{l}\text { Dilated ducts, focal enhancement } \\
\text { within lumen }\end{array}$ & $1(5.5)$ \\
\hline \multicolumn{2}{|l|}{ Kinetic curves: } \\
\hline Type 1 (early slow, persistent) & $1(5.5)$ \\
\hline Type 2 (early fast, plateau) & 11(61.1) \\
\hline Type 3(early wash-in, wash-out) & $6(33.3)$ \\
\hline Associated abnormal axillary lymph nodes & $4(22.2)$ \\
\hline
\end{tabular}

complaints of palpable lumps, nonspecific tenderness, and 2 of the 13 patients also had spontaneous unilateral nipple discharge.

On HPE, LCIS was incidentally seen in 14 patients with concurrent malignant lesions (4 of invasive lobular carcinoma, 4 of ductal carcinoma in situ (DCIS), and 6 of invasive ductal carcinoma). In two patients, LCIS was seen coexistent with benign lesions ( 1 of fibroadenoma and 1 of idiopathic granulomatous mastitis). These 16 cases were excluded for the analysis of the study.

Twenty-five cases of pure LCIS as the only pathologic abnormality on HPE were included for the study. All 25 cases who underwent DM with DBT showed abnormalities as mentioned in - Table 1. Supplemental USG was performed by the radiologist in all 25/25 (100\%) patients and no abnormality was detected in $11 / 25$ (44\%) cases. Dynamic CEMRI was done in 18/25 (72\%) cases in our study. The salient findings on breast imaging are described in -Table 2 .

\section{Imaging and Pathology Features}

LCIS is not usually associated with any clinical abnormality or imaging findings. ${ }^{7}$

Imaging methods are becoming increasingly sensitive, and the consequent detection of small lesions and subtle abnormalities increases the chance of detection of in situ carcinomas. $^{8}$

The findings on breast imaging of LCIS are varying and depend on the histologic type of the disease. It is part of a spectrum of abnormalities called lobular intraepithelial neoplasia. ${ }^{9}$ LCIS is considered as a marker of increased risk rather than a true precursor of lobular carcinoma. ${ }^{7}$

In this article, we describe a spectrum of imaging findings of LCIS in conjunction with its various pathologic correlates on mammography, USG, and dynamic CEMRI (-Table 3). It is important to understand that the histopathology dictates the radiological findings. The imaging and pathology correlation is essential for further management and follow-up.

Pure LCIS can be cLCIS or nonclassic LCIS types. cLCIS usually is occult on mammography. It is diagnosed on pathology as an incidental finding in core biopsy or surgical excision specimens in some patients who underwent excision for concurrent malignant lesions. ${ }^{1}$

Studies have shown an underestimation rate of LCIS ranging from 0 to $35 \% .^{8,10,11}$

LCIS is a proliferation centered in the TDLU, and consists of neoplastic cells that fill and expand most ( $>50 \%$ ) of the acini immunohistochemical stain for E-cadherin that is routinely used to distinguish lobular from ductal lesions, and is especially useful in separating LCIS variants from DCIS in cases with ambiguous morphology. Most cases of LCIS demonstrate complete loss of E-cadherin staining and diffuse cytoplasmic accumulation of p120. The results of immunohistochemical staining should always be interpreted in the context of morphologic findings (-Fig. 1).

cLCIS is usually positive for estrogen receptor and/ or progesterone receptor and negative for human epidermal growth factor receptor 2 status. ${ }^{1}$ 
554 Imaging Spectrum of LCIS and Correlation with Pathology Findings Hande et al.

Table 3 LCIS pathologic correlates on mammography, USG, and dynamic CEMRI

\begin{tabular}{|c|c|c|c|c|c|c|}
\hline A & B & C & D & $\mathrm{E}$ & $F$ & G \\
\hline & cLCIS & $\begin{array}{l}\text { cLCIS with } \\
\text { spherulosis }\end{array}$ & cLCIS with ALH & CLCIS with SA & pLCIS & ncLCIS \\
\hline \multicolumn{7}{|l|}{ Mammography } \\
\hline Negative findings & Common & Obscured & Obscured, rare & $\begin{array}{l}\text { Nonspecific } \\
\text { findings }\end{array}$ & $\begin{array}{l}\text { Nonspecific } \\
\text { findings }\end{array}$ & $\begin{array}{l}\text { Nonspecific } \\
\text { findings }\end{array}$ \\
\hline Mass & Rare & Usually absent & May be present & May be present & May be present & May be present \\
\hline Microcalcifications & May be present & Usually present & May be present & May be present & $\begin{array}{l}\text { Amorphous/ } \\
\text { pleomorphic }\end{array}$ & May be present \\
\hline $\begin{array}{l}\text { Architectural } \\
\text { distortion }\end{array}$ & May be present & May be present & May be present & Usually present & May be present & May be present \\
\hline Asymmetry & May be present & May be present & May be present & May be present & Usually present & May be present \\
\hline Associated finding & Usually absent & Usually absent & Usually absent & Usually absent & Rare, obscured & Rare, obscured \\
\hline \multicolumn{7}{|l|}{ Ultrasound } \\
\hline Mass & Rare & Rare & May be present & May be present & May be present & May be present \\
\hline Calcification & Obscured & May be present & Rare & May be present & May be present & Rare \\
\hline $\begin{array}{l}\text { Architectural } \\
\text { distortion }\end{array}$ & May be present & May be present & May be present & May be present & May be present & May be present \\
\hline Duct changes & May be seen & $\begin{array}{l}\text { Unassociated if } \\
\text { seen }\end{array}$ & $\begin{array}{l}\text { Unassociated if } \\
\text { seen }\end{array}$ & $\begin{array}{l}\text { Unassociated if } \\
\text { seen }\end{array}$ & May be present & May be present \\
\hline Vascularity & Rare & Rare & Rare & Variable & May be present & May be present \\
\hline Cystography & Variable & Not significant & Variable & Variable & Increased, hard & Variable \\
\hline Lymph nodes & Not significant & Not significant & Not significant & Not significant & Not significant & Not significant \\
\hline \multicolumn{7}{|l|}{ CEMRI } \\
\hline BPE & $\begin{array}{l}\text { Mild/moderate/ } \\
\text { severe }\end{array}$ & Mild/moderate & Moderate/severe & Moderate/severe & Moderate/severe & Moderate/severe \\
\hline Focus & May be present & Not significant & May be present & May be present & May be present & May be present \\
\hline $\begin{array}{l}\text { Enhancing irregular } \\
\text { mass }\end{array}$ & May be present & May be present & May be present & May be present & May be present & May be present \\
\hline $\begin{array}{l}\text { Nonmass } \\
\text { enhancement }\end{array}$ & Regional if present & May be present & May be present & May be present & May be present & May be present \\
\hline $\begin{array}{l}\text { Nonenhancing } \\
\text { lesion }\end{array}$ & May be present & May be present & May be present & May be present & May be present & May be present \\
\hline $\begin{array}{l}\text { Architectural } \\
\text { distortion }\end{array}$ & May be present & Rare & May be present & May be present & May be present & May be present \\
\hline Associated finding & Usually absent & Usually absent & Usually absent & Usually absent & May be present & May be present \\
\hline Kinetic curves & Type 2 or 3 & Type 2 & Type 2 or 3 & Type 2 or 3 & Type 2 or 3 & Type 2 or 3 \\
\hline Calcifications & Obscured isolated & Isolated rare & Obscured & $\begin{array}{l}\text { Associated with } \\
\text { mass }\end{array}$ & $\begin{array}{l}\text { Associated with } \\
\text { mass }\end{array}$ & $\begin{array}{l}\text { Associated with } \\
\text { mass }\end{array}$ \\
\hline
\end{tabular}

Abbreviations: ALH, atypical lobular hyperplasia; BPE, background parenchymal enhancement; CEMRI, contrast-enhanced computed tomography; LCIS, lobular carcinoma in situ; CLCIS, classic LCIS; ncLCIS, nonclassic LCIS; pLCIS, pleomorphic LCIS; SA, sclerosing adenosis; USG, ultrasonography.

CLCIS includes atypical lobular hyperplasia (ALH) as the cells composing ALH are morphologically indistinguishable from those of CLCIS, but the proliferation is limited to $<50 \%$ of the acini. ${ }^{1,11}$ ALH is associated with a four- to fivefold increase in the risk of subsequent breast carcinoma. Our study had ALH associated with two (8\%) cases.

cLCIS may involve collagenous spherulosis that is usually an incidental microscopic finding associated with myoepithelial hyperplasia. There may be only subtle focal asymmetry noted on the mammogram (-Fig. 2). These cases can be also be detected early due to associated calcifications that can be visible on mammography. ${ }^{12}$

CLCIS may coexist with sclerosing adenosis (SA) that is a benign sclerosing change of the TDLU, characterized by distortion of acini and glands and stromal sclerosis that can closely simulate invasive carcinoma. Myoepithelial hyperplasia and epithelial calcifications are common. cLCIS may involve SA that may be focal or diffuse and is often detected mammographically due to associated calcifications (-Fig. 3), architectural distortions, or an ill-defined mass 


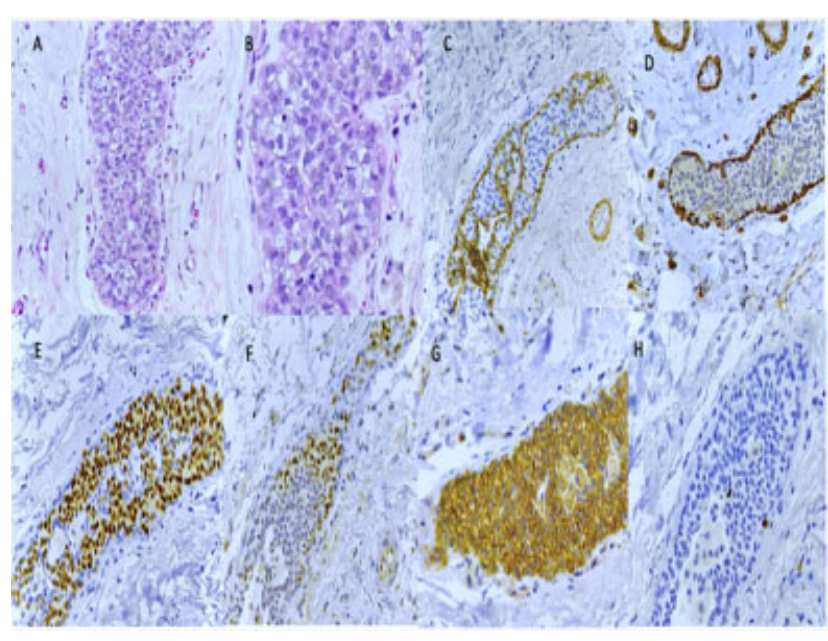

Fig. 1 Microscopy of classical lobular carcinoma in situ: (A) hematoxylin and eosin (H\&E) 20x. Solid proliferation of cells filling and dilating terminal duct lobular unit. (B) H\&E 40x. Individual cells are round monotonous in appearance; cellular atypia is mild to moderate. Immunohistochemistry: (C) E-cadherin. Intraluminal cells do not express E-cadherin, hallmark of lobular neoplasia. (D) Smooth muscle myosin (SMM). Positive staining of myoepithelial cells at the periphery of duct indicates this as in situ lesion. (E) Estrogen receptor: Neoplastic cells show strong nuclear expression of estrogen receptors. (F) Progesterone receptor: positive expression is seen in many of the cells. (G) Human epidermal growth factor receptor 2 Neu expression is equivocal $2+$. $(\mathrm{H}) \mathrm{Ki67}$ proliferation marker is low in this neoplasia.

lesion. ${ }^{1,13}$ SA lesions showed distortion or calcifications on mammograms, low echo-level nodules with heterogenous echo on USG and mass-like lesion with or without spiculated mass on CEMRI. ${ }^{13}$ In our study, three (12\%) cases showed associated SA with LCIS. Isolated architectural distortions with no associated microcalcifications were also seen in our study (-Fig. 4).

Pleomorphic LCIS (pLCIS) is LCIS variant where mitotic activity with marked nuclear pleomorphism is present. pLCIS closely mimics DCIS due to its solid growth pattern and there may be necrosis and calcifications. ${ }^{1}$ Immunohistochemical stains for E-cadherin and p120 can help resolve the differential diagnosis.

Mammography can show calcifications in up to 21 to $67 \%$ of cases. ${ }^{14,15}$ DBT thick slices with 2DSM revealed grouped pleomorphic or amorphous microcalcifications (-Fig. 5).

In our study, suspicious microcalcifications was seen in only $24 \%$ of cases and in $60 \%$ cases these were associated with other abnormalities, that is, mass (12\%), asymmetry (16\%) and architectural distortions (8\%) which is in concurrence with published studies. MRI, however, did not show microcalcifications as an isolated finding.

In our study, DBT images with $1 \mathrm{~mm}$ thin slices interpreted in conjunction with 2DSM and FFDM allow detection of architectural distortions (20\%) in addition to masses (24\%) and asymmetry (28\%).

cLCIS presenting as nodular lesions is not very common and has been described in studies by Stein et al and Christiano et $\mathrm{al}^{14,15}$ LCIS variants, of pLCIS and LCIS with central necrosis, are usually detected mammographically due to associated pleomorphic calcifications, or can present as a mass lesion with or without associated calcifications. ${ }^{16,17}$ In our study, mass with calcifications was found in three (12\%) cases (-Fig. 6). However, isolated nodular density or a mass was seen in three (12\%) cases ( $\mathbf{- F i g . ~} \mathbf{7}$ ).

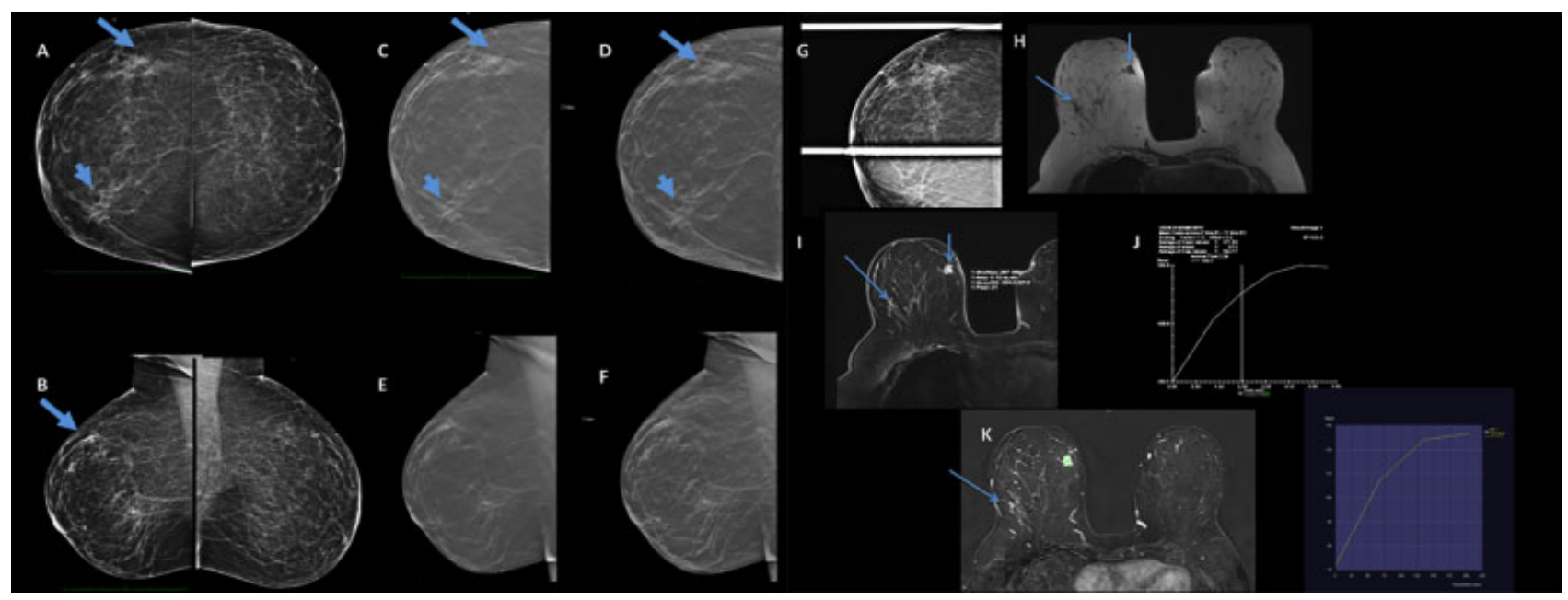

Fig. 2 Screening mammogram in a 60-year-old lady. Full-field digital mammography system ( $A$ and $B$ ) shows focal asymmetry in superolateral quadrant (long arrow) and in the medial aspect (arrowhead) of the right breast. Digital breast tomosynthesis (DBT) 1 mm tomosynthesis slices and two-dimensional synthesized mammography C-view images of right breast in craniocaudal (CC) (C and D) and mediolateral-oblique projections (E and F) BIRADS 4A. (G) Additional spot compression view in CC projection shows persistent focal asymmetry with no definite mass. Breast magnetic resonance imaging (MRI): (H) Axial T1-weighted image shows hypointense circumscribed mass medially (vertical arrow) and irregular hypointense lesion superolaterally (oblique arrow). Dynamic contrast-enhanced MRI shows ( $\mathrm{I}$ and $\mathrm{J}$ ) homogeneously enhancing lesion medially at 3 o'clock position (vertical arrow) with type 1 kinetic curve. (K) Heterogeneous nonmass enhancement with tiny enhancing focus laterally corresponding to focal asymmetry (oblique arrow) that showed type 2 curve on kinetics that was classic lobular carcinoma in situ on histopathological examination. 


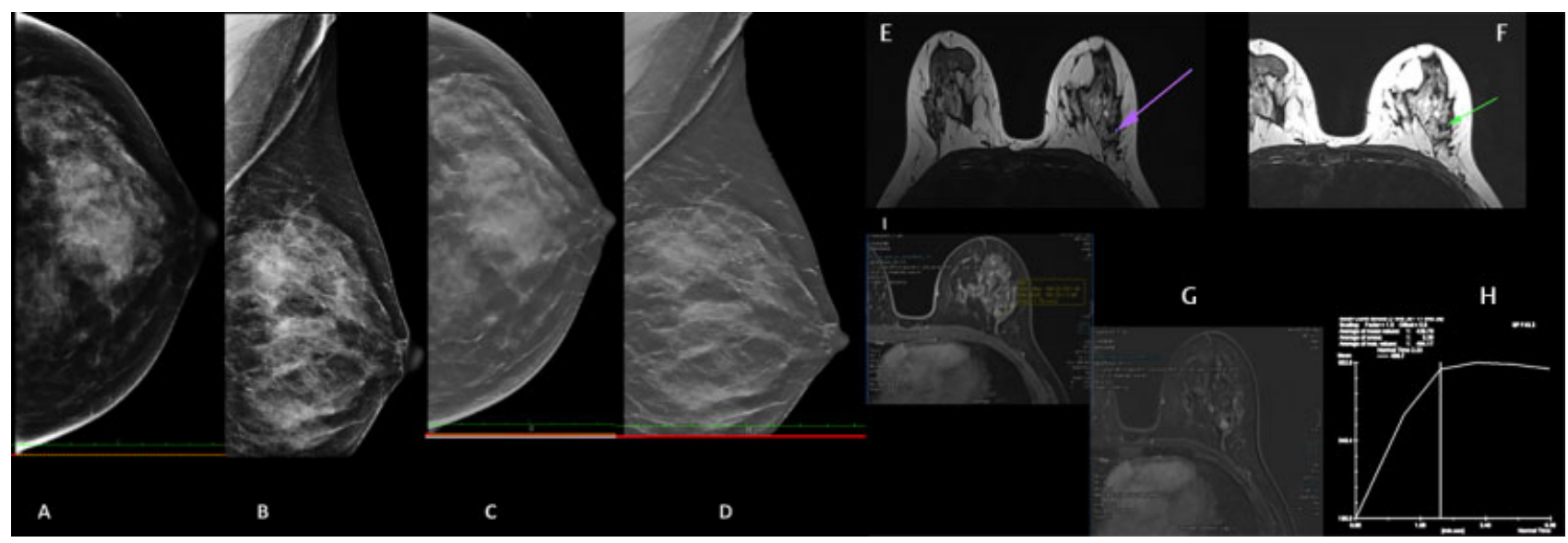

Fig. 3 Screening mammogram in a 51-year-old lady: (A and B) Left breast full-field digital mammography system craniocaudal (CC), mediolateral-oblique (MLO) views showing amorphous grouped microcalcifications with focal asymmetry in superolateral quadrant that is well visualized in digital breast tomosynthesis synthesized two-dimensional image (C-view) in (C) CC and (D) MLO projections BIRADS 4C. Magnetic resonance imaging (MRI) T1- and T2-weighted images: ( $E$ and F) hypointense focus in left breast (arrows). Dynamic contrast-enhanced MRI showed moderate background parenchymal enhancement with multiple enhancing foci. (G) Nonmass enhancement in the left breast 12 to 4 o'clock position. (H and I) Enhancing focus at 6 o'clock position in left breast with type 2 curve on kinetics.

CEMRI has a high sensitivity and enables evaluation of both breasts for multifocal and multicentric disease at the same time. Chu et al described that LCIS involves multicentric lobules in $85 \%$ and both the breasts in 30 to $67 \%$ of cases, respectively. ${ }^{3}$ MRI findings of LCIS are generally more subtle than those of DCIS or other invasive cancers.

In our study, of 18 cases who underwent dynamic CEMRI, the most common features were nonmass enhancement with heterogeneous enhancement in $8 / 18$ (44.4\%) cases. Enhancing foci $6 / 18$ (33.3\%) or mass with irregular margins and homogeneous enhancement $4 / 18(22.2 \%)$ were seen with moderate background parenchymal enhancement.

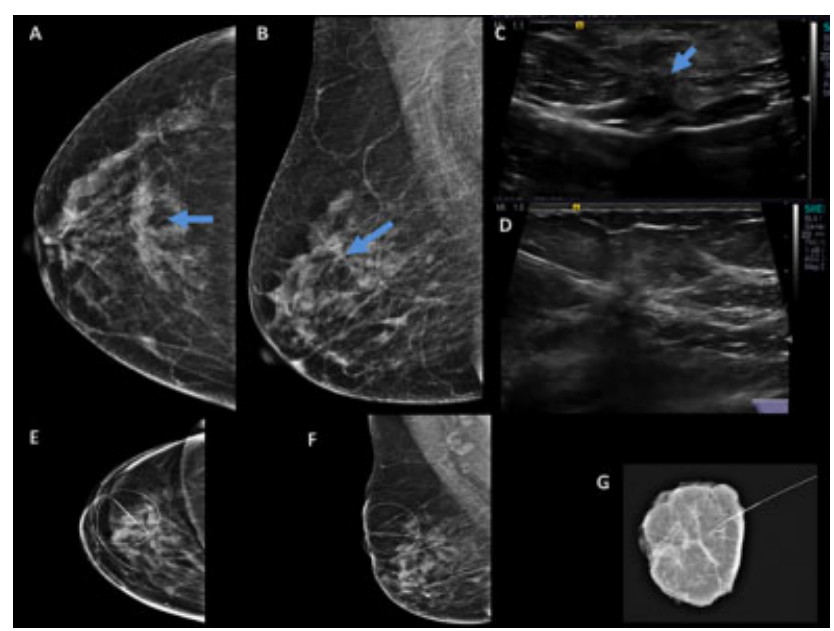

Fig. 4 Routine screening mammogram for a 50-year-old woman with a history of breast cancer in sister (first-degree relative): (A) Right breast full-field digital mammography system shows architectural distortion superiorly in craniocaudal (CC) view (straight arrow) and (B) mediolateral-oblique (MLO) view (angled arrow) well identified (C) ultrasonography (USG) shows architectural distortion at 12 o'clock position (long arrow). (D) Preoperative hook-wire localization done under USG guidance that is confirmed on mammogram to check the correct position in (E) CC and (F) MLO projections (G) specimen radiograph to confirm the excision of the lesion BIRADS 4B.
The kinetics of the lesions showed type 2 and type 3 early wash in- and wash-out curves most commonly.

Increased diagnosis due to widespread extensive breast screening because of increased awareness and age-specific incidence analysis revealed that the magnitude of the increase was highest among women aged $>50$ years; this age group is most likely to participate in routine mammographic screening. ${ }^{1}$ The age group of women in our study was 41 to 69 years with median age of 54 years.

Following radiologic-pathologic review, core biopsy results were considered discordant in 4 of 25 cases (16\%). The most common reason for discordance was a mass seen on imaging but no mass-forming entity found at histology ( 3 cases).

One patient with nipple discharge revealed dilated duct in the inferolateral quadrant on DBT $1 \mathrm{~mm}$ tomosynthesis slice and on USG dilated duct at 7 to 8 o'clock position with echogenic contents in the lumen ( - Fig. 8). CEMRI revealed ill-defined abnormal enhancement within a dilated duct prompted a biopsy and LCIS on HPE. However, sufficient explanation for the enhancement was not found on pathology.

LCIS shares similar gene expression profiling features with invasive lobular carcinoma. ${ }^{1,3}$ Similar mutation profile between LCIS and synchronous or metachronous invasive carcinoma was found in various studies, supporting the role of LCIS as a risk factor and nonobligate precursor to invasive carcinoma. ${ }^{1,18,19}$ The National Comprehensive Cancer Network guidelines currently recommend excisional biopsy in LCIS cases.

\section{Implications and Key Messages}

LCIS is frequently mammographically occult, and an incidental finding on routine screening mammograms, usually because of microcalcifications. ${ }^{3}$ Technical advances with better resolution result in higher sensitivity of imaging modalities. This helps in increased probability of detection of subtle abnormalities on screening mammograms. In our 


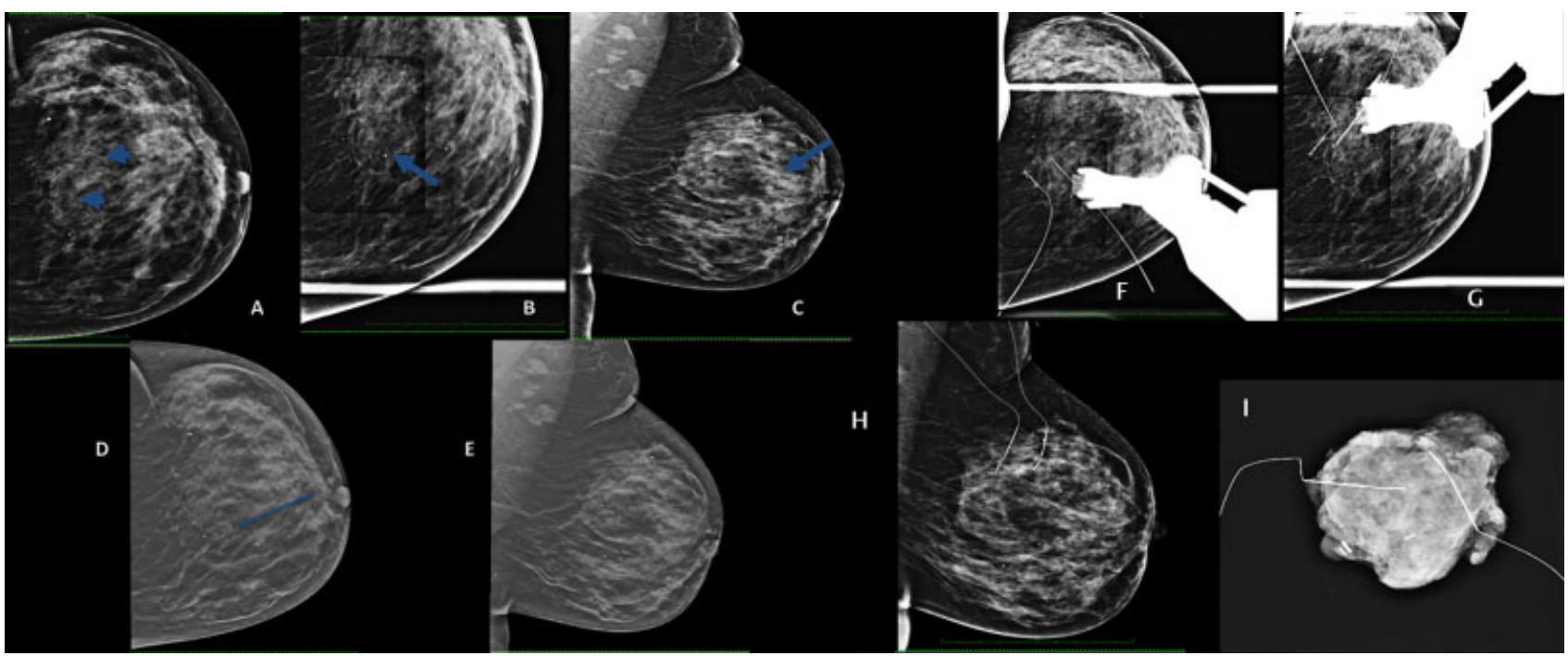

Fig. 5 Screening mammogram in a 54-year-old woman: (A) Left breast reveals suspicious group microcalcifications (arrow) in superolateral quadrant. (B) Additional spot magnification view shows pleomorphic and few amorphous microcalcifications in linear distribution seen on (C) mediolateral-oblique (MLO) view; well appreciated in (D and E) craniocaudal (CC) and MLO tomosynthesis slices. Breast imaging reporting BIRADS 4C (F and G) Preoperative stereotactic mammography-guided hook-wires placed in CC position with bracketing for microcalcifications. (H) Preoperative stereotactic mammography-guided 2 hook-wires placed with bracketing confirmed in place in ML projection. (I) Specimen radiograph of the excised microcalcifications.

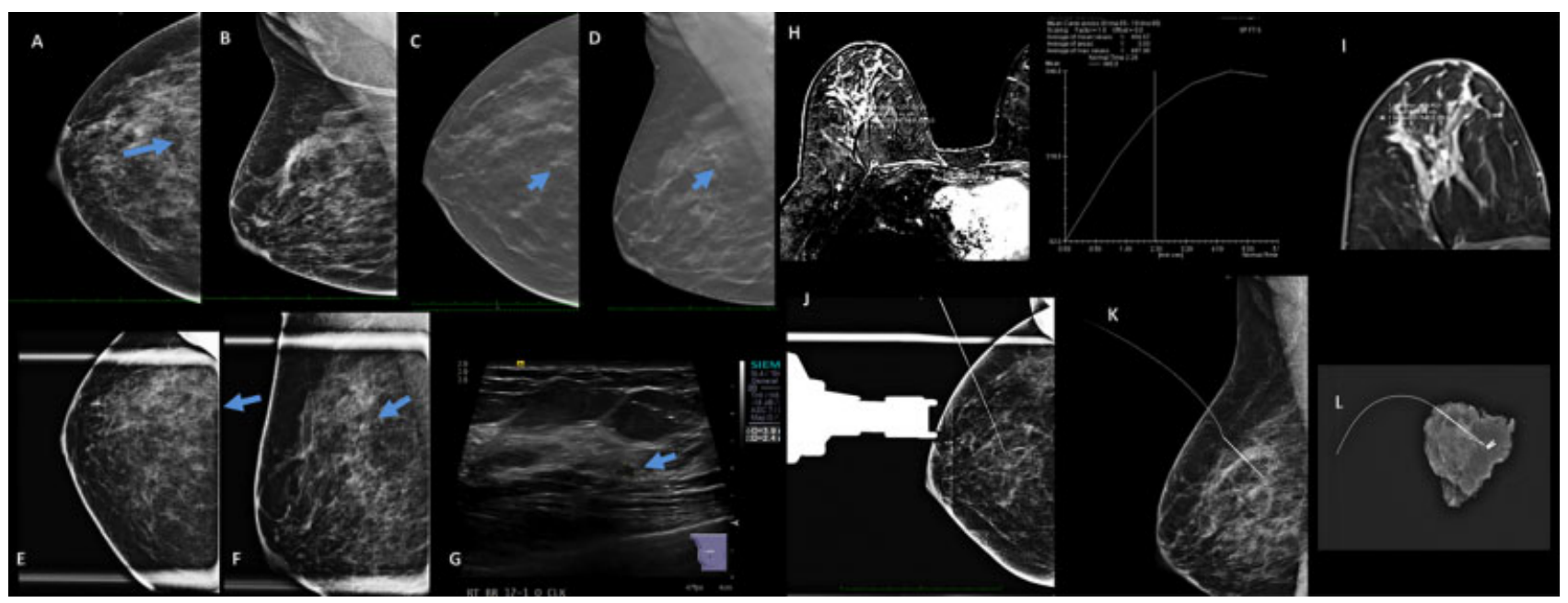

Fig. 6 Screening mammography in a 53-year-old lady: (A and B) full-field digital mammography system right breast reveals irregular focal density in craniocaudal (CC) and mediolateral-oblique (MLO) views posteriorly that are well appreciated on (C and D) tomosynthesis $1 \mathrm{~mm}$ slices with spiculated margins and faint microcalcifications within (arrowheads) (E and F). Additional spot compression views in CC, MLO projections. (G) High-resolution ultrasonography reveals a tiny hypoechoic oval lesion. BIRADS 4C. (H and I) Dynamic contrast-enhanced magnetic resonance imaging (MRI) axial T1-weighted shows tiny enhancing lesion in postcontrast sequence with type 2 kinetic curve in the right breast corresponding to the area with microcalcifications which are appreciated in magnetic resonance imaging. ( $\mathrm{J}$ and $\mathrm{K}$ ) Stereotactic mammographyguided hook-wire localization for the lesion in right breast. (I) Radiograph of surgical specimen excised.

study, DBT images with thin slices of up to $1 \mathrm{~mm}$ interpreted in conjunction with 2DSM and FFDM allow detection of architectural distortions better (-Fig. 9) in addition to masses and asymmetry.

LCIS can sometimes mimic invasive carcinoma on USG with a suspicious mass lesion with irregular margins and adjunctive CEMRI enables a more confident reporting.

Image-guided needle biopsy is routinely performed for suspicious BI-RADS 4, 5 lesions and followed by excision as recommended. The diagnosis is confirmed by HPE and IHC examinations.
Follow-up and close surveillance with routine annual breast screening with mammography, USG, and if required MRI as an adjunct modality especially in dense breasts are recommended in women diagnosed with LCIS. ${ }^{2,5}$

The patients in our study group were followed up for 7 to 65 months and no recurrence was detected on breast imaging. One patient reported with complaint of palpable lump at the surgical site, 18 months after excision of a suspicious architectural distortion detected as on the initial screening mammogram. Follow-up mammography revealed a focal high-density mass that was seen as an oval, circumscribed 


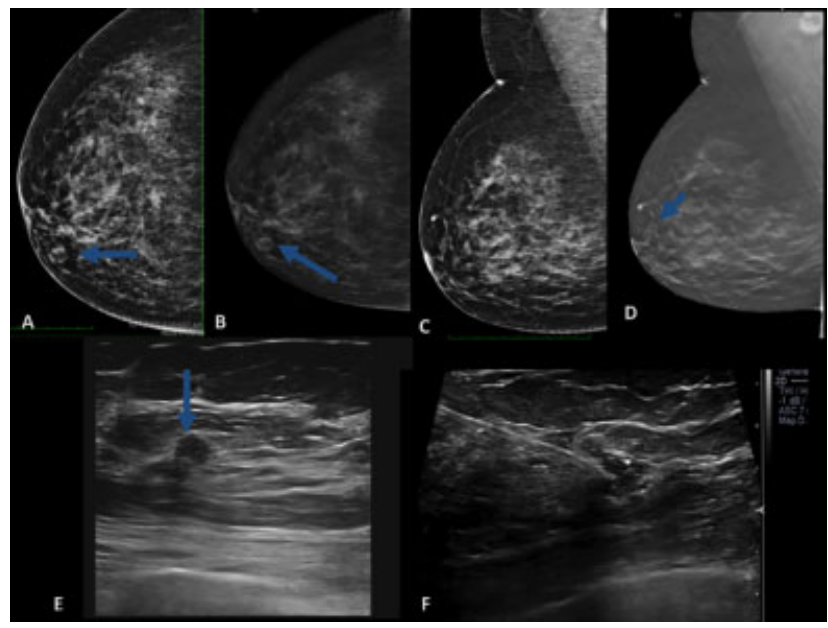

Fig. 7 Mammography in a 52-year-old lady with a family history of breast cancer in mother with palpable lump in right breast: (A) fullfield digital mammography system craniocaudal view shows a rounded focal density in retroareolar region medially in right breast (arrow), well appreciated in (B) $1 \mathrm{~mm}$ tomosynthesis slice 33/69 (angled arrow). (C) Standard mediolateral-oblique (MLO) view does not show the lesion that is well identified in (D) $1 \mathrm{~mm}$ tomosynthesis $62 / 93$ in MLO projection (short thick arrow). (E) Ultrasonography (USG) shows oval, circumscribed hypoechoic lesion with angular margins at 10 o'clock position (vertical arrow) in the left breast. BIRADS 4C. (F) USG-guided core biopsy performed with 14 G needle.

hypoechoic lesion on USG. CEMRI revealed this as a hyperintense lesion on T2-weighted imaging with hypointense rim at the site of scar with no enhancement of the mass on postcontrast images. USG-guided core biopsy revealed fat necrosis with benign postoperative changes (-Fig. 10). The patient has been on routine short-term follow-up every

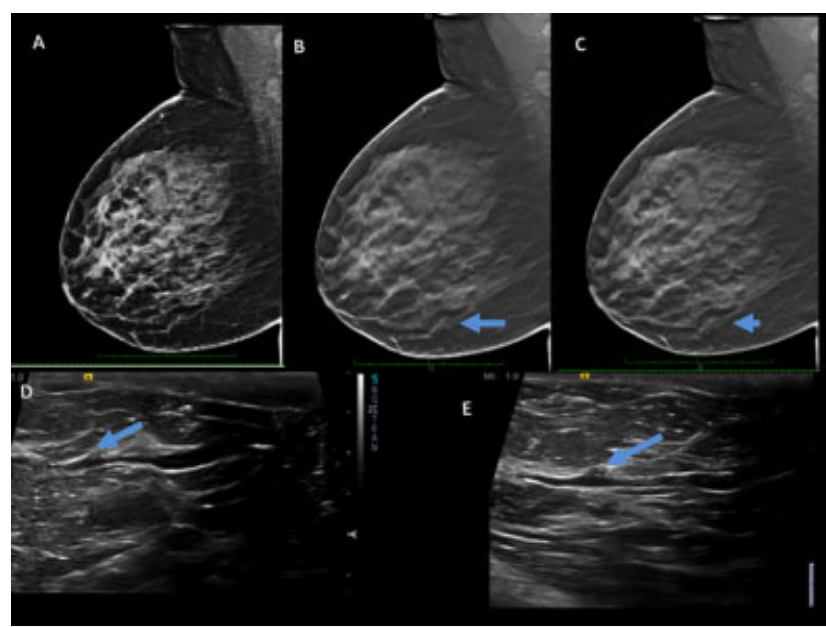

Fig. 8 Mammography in a 54-year-old lady with a history of spontaneous nipple discharge from right breast (A) full-field digital mammography system in mediolateral-oblique (MLO) view shows dense breast with no obvious abnormality. (B) $1 \mathrm{~mm}$ tomosynthesis slice reveals dilated duct in the inferolateral quadrant (arrow) which is well identified in (C) 2D synthesized C-view image (short arrow) in MLO. (D and E) Dilated duct at 7 to 8 o'clock position (angled arrow) with echogenic contents in the lumen (long arrow) on ultrasonography BIRADS $4 \mathrm{~B}$.

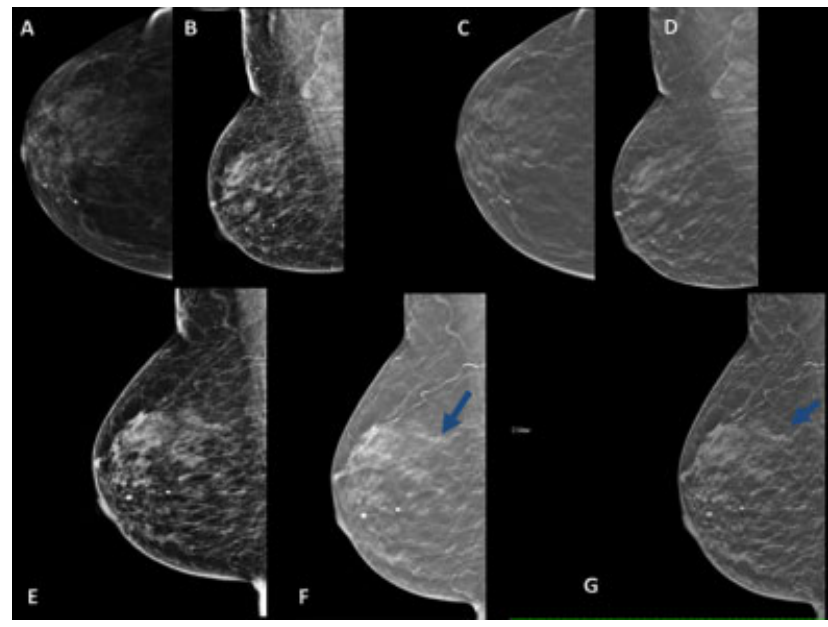

Fig. 9 Left mastectomy status for carcinoma breast: Postoperative follow-up annual mammography. (A and B) Full-field digital mammography system (FFDM) of right breast in craniocaudal (CC) and mediolateral-oblique (MLO) views shows focal asymmetry in superolateral quadrant. Benign coarse calcifications of liponecrosis and vascular calcifications are noted. Digital breast tomosynthesis (DBT) $1 \mathrm{~mm}$ slices in (C) in CC show no obvious abnormality. (E) FFDM in ML view shows architectural distortion posteriorly which is well appreciated in ( $D$ and $F) 1 \mathrm{~mm}$ tomosynthesis slices in MLO and ML projections and in $(G)$ DBT two-dimensional synthesized image. BIRADS 4B.

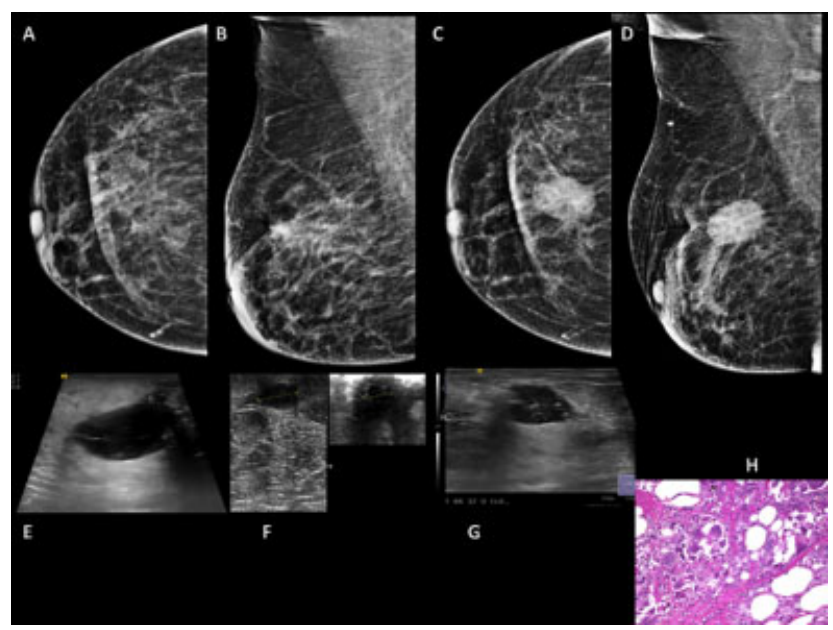

Fig. 10 Lobular carcinoma in situ on regular surveillance. Postoperative follow-up mammogram of patient (illustrated case 4). Full-field digital mammography system (FFDM) (A and B) in craniocaudal and mediolateral-oblique views after 12 months shows postsurgical changes with architectural distortion; 18 months later presented with palpable lump at the site of scar. FFDM shows (C and D) oval, focal density with circumscribed margins associated with architectural distortion of scar tissue. Ultrasonography (USG) shows (E) wellcircumscribed, oval hypoechoic lesion with angular margins and shadowing over the palpable mass at the postsurgical scar site (F) that was seen as firm to hard on elastography. (G) USG-guided core biopsy from the well circumscribed, oval hypoechoic lesion was done. $(\mathrm{H})$ Biopsy histopathological examination showed features suggestive of fat necrosis. 
6 months with USG and annual mammogram for the past 28 months with no new findings.

\section{Conclusion}

Pure LCIS is currently recognized as an intermediate risk factor for subsequent development of invasive breast cancer of up to 7 to 12 times increased risk in women with LCIS as compared with those without ${ }^{3}$ and 9 to 10 times that of general population after the diagnosis of cLCIS. ${ }^{1}$ It is important that the radiologist must recognize and detect the varied imaging appearances of this entity. This directly impacts the management and follow-up of LCIS confirmed on HPE and IHC that affects the future outcome of these cases.

Conflicts of Interest

There are no conflicts of interest.

Financial Support and Sponsorship

Nil.

\section{References}

1 Wen HY, Brogi E. Lobular carcinoma in situ. Surg Pathol Clin 2018; 11(01):123-145

2 Schwartz T, Cyr A, Margenthaler J. Screening breast magnetic resonance imaging in women with atypia or lobular carcinoma in situ. J Surg Res 2015;193(02):519-522

3 Chu AJ, Cho N, Park IA, Cho SW. Features of pure lobular carcinoma in situ on magnetic resonance imaging associated with immediate reexcision after lumpectomy. J Breast Cancer 2016;19(02):199-205

4 Arpino G, Laucirica R, Elledge RM. Premalignant and in situ breast disease: biology and clinical implications. Ann Intern Med 2005; 143(06):446-457

5 Sung JS, Malak SF, Bajaj P, Alis R, Dershaw DD, Morris EA. Screening breast MR imaging in women with a history of lobular carcinoma in situ. Radiology 2011;261(02):414-420

6 D'Orsi C, Sickles EA, Mendelson EB, Morris EABreast Imaging Reporting and Data System: ACR BI-RADS Breast Imaging Atlas. 5th edition. Reston, VA: American College of Radiology2013
7 Chaudhary S, Lawrence L, McGinty G, Kostroff K, Bhuiya T. Classic lobular neoplasia on core biopsy: a clinical and radio-pathologic correlation study with follow-up excision biopsy. Mod Pathol 2013;26(06):762-771

8 Graziano L, Bitencourt AGV, Guatelli CSet al.Lobular carcinoma in situ with atypical mass presentation: a case report. Rev Bras Ginecol Obstet 2016;38(02):112-116

9 Jorns J, Sabel MS, Pang JC. Lobular neoplasia: morphology and management. Arch Pathol Lab Med 2014;138(10):1344-1349

10 Zhao C, Desouki MM, Florea A, Mohammed K, Li X, Dabbs D. Pathologic findings of follow-up surgical excision for lobular neoplasia on breast core biopsy performed for calcification. Am J Clin Pathol 2012;138(01):72-78

11 Murray MP, Luedtke C, Liberman L, Nehhozina T, Akram M, Brogi E. Classic lobular carcinoma in situ and atypical lobular hyperplasia at percutaneous breast core biopsy: outcomes of prospective excision. Cancer 2013;119(05): 1073-1079

12 Eisenberg RE, Hoda SA. Lobular carcinoma in situ with collagenous spherulosis: clinicopathologic characteristics of 38 cases. Breast J 2014;20(04):440-441

13 Tan H, Zhang H, Lei Z, Fu F, Wang M. Radiological and clinical findings in sclerosing adenosis of the breast. Medicine (Baltimore 2019;98(39):e17061

14 Stein LF, Zisman G, Rapelyea JA, Schwartz AM, Abell B, Brem RF. Lobular carcinoma in situ of the breast presenting as a mass. AJR Am J Roentgenol 2005;184(06):1799-1801

15 Christiano JG, Duncan LD, Bell JL. Lobular carcinoma in situ of the breast presenting as a discrete mass. Am Surg 2012;78(01): E38-E40

16 Maxwell AJ, Clements K, Dodwell DJ, et al.Sloane Project Steering Group. The radiological features, diagnosis and management of screen-detected lobular neoplasia of the breast: findings from the Sloane Project. Breast 2016;27:109-115

17 Flanagan MR, Rendi MH, Calhoun KE, Anderson BO, Javid SH. Pleomorphic lobular carcinoma in situ: Radiologic-pathologic features and clinical management. Ann Surg Oncol 2015;22 (13):4263-4269

18 Hwang ES, Nyante SJ, Yi Chen Yet al.Clonality of lobular carcinoma in situ and synchronous invasive lobular carcinoma. Cancer 2004; 100(12):2562-2572

19 Begg CB, Ostrovnaya I, Carniello JVet al.Clonal relationships between lobular carcinoma in situ and other breast malignancies. Breast Cancer Res 2016;18(01):66 